Cite as: Howells, A., Ivtzan, I., \& Eiroa-Orosa, F. J. (2016). Putting the "app" in Happiness: A Randomised Controlled Trial of a Smartphone-Based Mindfulness Intervention to Enhance Wellbeing. Journal of Happiness Studies, 17(1), 163-185. https://doi.org/10.1007/s10902-014-9589-1

\title{
"Putting the 'app' in happiness: A randomised controlled trial of a smartphone-based mindfulness intervention to enhance wellbeing."
}

Annika Howells, Itai Ivtzan, Francisco Jose Eiroa-Orosa

University Of East London, Stratford Campus, Water Lane, London, E15 4lz.

Contact: $\underline{\text { howells.annika@gmail.com, }}+61424932363$ 


\begin{abstract}
Smartphones are revolutionizing approaches to wellbeing investment. Those seeking greater happiness can engage with thousands of downloadable self-help applications instantly, yet the effectiveness of these strategies remains unknown. As wellbeing can be significantly enhanced by positive psychological interventions, the present investigation explored the viability of delivering this content in application format to the happiness seekers it is targeting. A smartphone-based randomized-controlled trial was conducted with a self-selecting pool, randomly assigned to engage with an empirically based mindfulness intervention $(n=57)$ or a placebo intervention $(n=64)$ for ten days. The study explored the feasibility of smartphone intervention delivery, the importance of empirically based content and the extent to which subjective experience ratings related to wellbeing gains. Results showed engagement with the mindfulness application lead to significant gains in positive affect with a medium effect size and reduced depressive symptoms with a small effect size. No significant gains were observed in the control condition. Ratings of task enjoyment were positively associated with wellbeing gains. A positive trend was observed between task ease and gains made. Findings support the viability of smartphone-based interventions to significantly enhance wellbeing, underscoring the importance of application content and person-activity fit. This investigation presents implications for happiness seeking strategies in the real world whilst showcasing a dynamic method of intervention delivery that can benefit future research and practice. The greatest mission of positive psychology is to enhance global flourishing; the prevalence of smartphones and the potential of interventions may play a vital role.
\end{abstract}

Keywords: happiness; mindfulness; wellbeing; positive psychological intervention; randomized controlled trial; smartphone application. 


\section{Introduction}

\section{Psychology and the Smartphone Revolution}

We live in an age where technology is constantly evolving; becoming rapidly smarter, better and faster. The technological revolution of the twenty-first century has infiltrated all aspects of daily life, affecting thoughts, behaviour, consumption and social interactions in new and unexpected ways. Smartphones have played an important role in this revolution. An estimated 1.82 billion active smartphones were in use globally by the end of 2013 (Khalaf, 2013).

Smartphone devices are used for much more than communicating; the vast functionality leaves little that a smartphone can't do. This diversity has created unprecedented user dependency. Devices are checked on average every 6.5 minutes, or 150 times per day and users spend most of their time engaging with applications (Khalaf, 2013). The astounding rise of the smartphone has prompted compelling calls for this method to be more widely used within psychological research (Intille, 2012; Eonta et al., 2011; Raento, Oulasvirta \& Eagle, 2009). As well as being highly accessible, versatile and cost effective, they take studies far beyond the confines of laboratory-based studies with student populations, and into the real world (Miller, 2012). Dufau et al. (2011, p.1) reason that this methodology "has the potential to uncover laws of mind that have previously been hidden in the noise of small-scale experiments", or as Miller (2012, p.221) says - they are "an occasion to rethink what psychology could be".

Discernable links between smartphone technology and positive health outcomes have already been recognized in many fields, with preventative health and clinical interventions leading the way (Stevens \& Bryan, 2012; Ozdalga, Ozdalga \& Ahuja, 2012; Hebden, Cook, Van der Ploeg \& Allman-Farinelli, 2012; Boulos, Wheeler, Tavares \& Jones, 2011; Luxton, McCann, Bush, Mishkind \& Reger, 2011; Busis, 2010; Wolfenden, Brennan \& Britton 2010). 
Within psychology, mobile devices have been used in addiction treatment (McTavish, Chih, Shah \& Gustafson, 2012), to accompany the delivery of therapeutic interventions (Ly, Dahl, Carlbring, \& Andersson, 2012; Clough \& Casey, 2011) and to generate rich sets of real world behavioural data (Dufau et al., 2011, Killingsworth \& Gilbert 2010). Positive psychology specifically has gained from Parks, Della Porter, Pierce, Zilca and Lyubomirsky's (2012) use of smartphone-based methodology offering application-based strategies to boost happiness. Their study not only revealed new insights into intervention engagement, smartphone methodology and those pursuing happiness; studying participants in this naturalistic way markedly enhanced the ecological validity of the findings and replaced prior assumptions with empirical findings.

\section{Seeking Happiness - "There's an app for that"}

Happiness is undeniably a personal investment worthy of pursuit. It is reputed to generate life successes as well as confirm their existence (Lyubomirsky, King \& Diener, 2005). Beyond hedonistic pleasure seeking, meta-analysis has shown subjectively rated wellbeing to be associated with multiple personal benefits such as longer life expectancy, higher earnings, more friends and a happier marriage (Lyubomirsky et al., 2005). "Happiness seekers" are those who make an intentional choice to pursue happiness and invest in their subjective wellbeing through the selection of strategies. These features make them the target population for all manner of happiness-enhancing interventions, including those researched and developed within the field of positive psychology (Parks et al., 2012). Yet happiness seekers have seldom been engaged in intervention research, and their characteristics, behaviours and needs have been largely presumed throughout the literature (Parks et al., 2012). Parks et al. (2012, Study 3) studied the preferences and practices of real world happiness seekers using smartphonebased methodology; specifically, analysing their usage of an application that delivered a suite of empirically based interventions. The study revealed important findings about happiness 
seeking practices and the potential to enhance wellbeing through an application. These insightful and highly ecologically valid findings highlight the potential for further intervention research to engage diverse, real-world populations using smartphones. The popularity of such innovative methodology is likely to transpire within the coming years as real-world research methods enable psychological investigation to connect to the 21 st century.

In just five years since the introduction of smartphone applications, there are now more than 1.5 million available for download and by 2015 more than 89 billion applications will have been downloaded (Khalaf, 2013). The widest variety of strategies available to the millions of real-world happiness seekers is via smartphone based self-help applications. Tens of thousands are listed under "self-development", "self-help", "happiness" and "wellbeing", with content varying from gratitude journaling and coaching to providing support with clinical disorders - all downloadable to the device within seconds. Smartphone applications are much more accessible, diverse, flexible, interactive, dynamic, discreet and cheaper (many are free) than other available forms of self-development; cumulatively enhancing their appeal and popularity. The volume of content and instant accessibility signifies a supply to meet the demand created by mass interest in personal development originating from the self-help movement and the growing interest in positive psychology.

\section{Differentiating Between Helping Yourself and Self-Help}

Research originating from the field of positive psychology convincingly shows that a substantial portion of happiness is within an individual's control; through intentional activities and responses (Lyubomirsky, Sheldon, \& Schkade, 2005). Researchers have demonstrated that an assortment of brief, simple cognitive and behavioral strategies collectively known as positive interventions can effectively improve happiness and wellbeing (for detailed reviews see Layous \& Lyubomirsky, 2014; Parks and Biswas-Diener, 2013; Sin \& Lyubomirsky, 2009). These reviews feature diverse empirically based strategies such as participants 
expressing gratitude, performing acts of kindness, visualizing their best possible self, using character strengths and practicing mindfulness. Randomized controlled experiments have been used to establish that positive interventions are much more than placebos, signifying instead that an empirical basis is critical in markedly enhancing wellbeing over time (e.g. Layous, Nelson, \& Lyubomirsky, 2012; Boehm, Lyubomirsky, \& Sheldon, 2011a; Seligman, Steen, Park \& Peterson, 2005; Emmons \& McCullough, 2003).

Sin and Lyubomirsky (2009) conducted the first meta-analysis of positive interventions, demonstrating they could significantly enhance wellbeing and also decrease depressive symptoms. A meta-analysis of behavioural activation (BA) interventions for wellbeing by Mazzucchelli, Kane and Rees (2010) found comparable results. Most recently, Bolier et al. (2013) found the effect size of positive interventions to be small, but sustainable at follow up periods of three to six months. These outcomes collectively validate the science of wellbeing as incredibly worthwhile. Positive interventions demonstrate a developing understanding of human flourishing and practical ways this experience can be enhanced; both outcomes the field of positive psychology has aspired to achieve since formation (Seligman \& Csikszentmihalyi, 2000). Furthermore, these findings distinguish positive interventions from non-researched self-help. They are founding an empirically based dimension to happiness seeking, at a time when personal investment is still in such great demand (La Rosa, 2013). The science of positive psychology is able to propose rigorously tested, meaningful and sustainable ways to enhance wellbeing that would offer real-world happiness seekers a more rewarding and effective experience of helping themselves. To effectively reach this target audience, however, the delivery format must be characteristic of their usual practices (Parks et al., 2012), such as the development of content that is smartphone-based.

Regardless of influential endorsements and grandiose claims, the majority of strategies 
happiness seekers engage with are not supported by any form of research (Bergsma, 2008; Rosen, 1993, 1987). An astonishing lack of regulation and evidence base means very few of the 'happiness-boosting' self-help techniques available boast demonstrable effects. Whilst it is possible that they could be highly effective regardless of any scientific support; it is also plausible that they could be placebos, or indeed harmful. This rigorous scientific view highlights a critical dissonance between what happiness seekers believe they are engaging with, and what they are actually engaging with. That is, are individuals actually helping themselves by engaging with self-help?

Happiness seekers are motivated to become happier and spend time and resources purposefully striving to reach this goal (Parks et al., 2012, Study 2 and 3). The booming success of the non-researched self-help industry suggests that applying this mindset to engaging with strategies that don't have an empirical basis may enhance wellbeing to some extent. Individuals choosing (i.e. self-selecting) to use these forms of intervention may experience some slight gains in wellbeing resulting from positive illusions or a self-fulfilling prophecy (Kunda, 1990), regardless of the content, as they possess the will to become happier. Sin and Lyubomirsky (2009) identified self-selection as a possible moderator of positive intervention successes because those electing to participate in positive interventions experienced greater wellbeing gains, which may have related to their initial expectations (e.g Seligman, et al., 2005). Subsequent laboratory-based studies found that motivation to become happier was beneficial, but was not enough to significantly enhance wellbeing alone; empirical content was also necessary (Lyubomirsky, Dickerhoof, Boehm \& Sheldon, 2011). For example, Ferguson and Sheldon (2013, Study 2) found intention to become happier facilitated the outcome of a wellbeing enhancing exercise, leading to greater gains in happiness.

Ultimately the interaction of a combination of person and activity factors will determine 
the effectiveness of a positive intervention. Emerging research in this domain suggests that degree of 'fit' between the person and activity as captured by the user's experience is linked to gains in wellbeing (Layous \& Lyubomirsky, 2014). In a previous study, Schueller, (2010) found participants who indicated a preference for certain positive activities (i.e. enjoyed them, perceived a benefit from completing them and did not find them difficult) were more likely to follow those activities and ultimately showed greater increases in wellbeing. Subsequently, Schueller (2011) used user preference to match people to positive activities, with results demonstrating greater gains in wellbeing compared to the non-matched counterparts. Collectively, the available research on self-selection and fit suggests that individuals who intentionally seek to be happier, (i.e. have motivation and believe the strategies they use will work), when engaging with empirically-based content that fits their preferences, should experience the greatest wellbeing gains.

\section{Theory and Practice}

Over a decade of research into intervention efficacy has informed the development of the Positive Activity Model by Lyubomirsky and Layous (2013). Addressing how the features of both the activity and person can influence the success of positive activities as they are being performed, the model also accounts for how they unite to form the degree of fit. Therefore, it provides a suitable framework to guide the present investigation. Notably, this model encapsulates the remarkable progress of intervention literature and the science of wellbeing within a short time; from claims that trying to enhance happiness was futile given its genetic predisposition (Lykken \& Tellegen, 1996), to asking how happiness can be sustainably increased (Lyubomirsky et al., 2005), to identifying potential underlying mechanisms and optimal conditions leading to significantly enhanced wellbeing (Lyubomirsky \& Layous, 2013).

Whilst there is now sufficient theory supporting the notion of positive psychological 
interventions to enhance wellbeing, it is evident that there is a distinct lack of theoretical framework to guide the use of mobile technology to deliver these interventions. Mohr, Burns, Schueller, Clarke and Klinkman (2013) have examined the diversity of behavioural intervention technologies (BITs) used to create changes in the field of health. In recent years, BITs have been used to deploy a wide range of behaviour change strategies via a gamut of technology platforms from videoconferencing to gaming to smartphone devices. Yet Mohr et al. (2013) note how BITs have predominantly developed by adapting established paradigms, and the limitations this approach can bring. Whilst acknowledging the importance of current theoretical models derived from behavioural and psychological origins; what's missing is theory so dynamic that it can be applied to technology as it continues to develop. This could be feasibly created by taking an interdisciplinary approach (Dulin, Gonzalez, King, Giroux \& Bacon, 2013; Webb, Joseph, Yardley \& Mitchie, 2010). Mohr et al. (2013) recommend assimilating existing theory with more progressive models, for example, from control systems engineering. A merge of this nature would generate theoretical models as adaptive and dynamic as the practice it seeks to explore and develop.

\section{The Present Study}

Advances in mobile technology provide the means to investigate life as it is lived in innovative ways. It can connect research to real world practices on a global scale, yet the dynamic opportunities afforded by these methodologies are yet to be fully realized. Despite a substantial body of work on the efficacy of wellbeing enhancing interventions, the majority of research fails to engage those whom the interventions are predominantly targeting, or deliver activities in ways comparable to real world experiences. As happiness seekers continue to engage with non-researched interventions, the field of positive psychology demonstrates how interventions yield sizeable and sustainable effects. Herein lies the incongruence between experimental research and real world practice; where the happiness seeking experience could 
be greatly enriched and sustained whilst fulfilling a fundamental mission of positive psychology - to help individuals to flourish (Seligman, 2011).

To address these limitations, the present study was designed with innovation and relevance to the real world in mind. A global and diverse sample of self-selected happiness seekers was engaged to take part in an investigation more reflective of their natural practices by delivering intervention content through a smartphone application. The research involved a randomized-control trial of a mindfulness intervention delivered via smartphone in the first study of its kind.

\section{The Science of Mindfulness.}

The present investigation selected to use a mindfulness-based intervention for a number of key reasons. As one of many empirically based interventions to choose from, an application delivering mindfulness training was sourced primarily for its capacity to meet refined positive intervention criteria as outlined by Parks and Biswas-Diener (2013). The primary goal of the intervention was to induce positive outcomes, it was grounded in empirical findings, and it was appropriate for the population engaged. Secondly, meditation was identified in a previous study as the least practiced intervention strategy by real world happiness seekers (Parks et al., 2012, Study 2), indicating this type of intervention was less likely to be previously rehearsed, or currently practiced by the current sample.

Mindfulness is a practice of non-judgmental awareness of present moment experiences (Kabat-Zinn, 2005; see also Hart, Ivtzan, \& Hart, 2013) that is inherently linked to greater wellbeing. There is a wealth of scientific literature spanning more than three decades demonstrating the beneficial impact mindfulness meditation has on health, wellbeing and psychological functioning in both clinical and non-clinical populations (for reviews see Keng, Smoski \& Robins, 2011; Shapiro \& Carlson, 2009; Baer, 2003; Dillbeck \& Orme-Johnson, 1987). By researching long-term meditators and delivering mindfulness-based training to 
novice meditators, scientists are building a deeper understanding of the multiple pathways influenced by the practice that lead to greater psychological health (Baer, Lykins \& Peters, 2012; Brown, Ryan \& Creswell, 2007).

Remarkably, mindfulness interventions as brief as three short sessions have yielded positive outcomes (Harnett et al., 2010; Zeidan, Gordon, Merchant \& Goolkasian, 2010; see also Carmody \& Baer, 2009), and under certain conditions - instant gains have been reported (Ussher et al., 2014). Mindfulness-based training is predominantly delivered face to face, with a small number of studies trialling content delivered online (e.g. Cavanagh et al., 2013; Glück \& Maercker, 2011). The scarcity of smartphone-based mindfulness studies and no randomized controlled studies of this kind to date means the feasibility and potential of this format of intervention delivery remains largely unknown (Plaza, Demarzo, Herrera-Mercadal \& GarcíaCampayo, 2013).

\section{Hypothesis 1: Real content, real world, real change?}

Our first hypothesis concerned the role of empirically based content. Self-selected participants were randomly allocated to an experimental or control condition to determine whether having the motivation to become happier and then engaging with a mindfulness-based positive intervention would enhance wellbeing significantly more than a placebo control task. It was hypothesised that engaging with the positive intervention would significantly enhance participant wellbeing levels. An incorporated aim of this hypothesis was to support initial findings of Parks et al. (2012, Study 3); that smartphones are a valid platform to effectively deliver positive interventions.

\section{Hypothesis 2 and 3: Exploring the Smartphone Experience}

Our second and third hypothesis concerned the role of person-activity fit. As a new 
platform of intervention delivery, many factors remained unknown about smartphone-based delivery and experience, yet could be vital in its future usage and success. Guided by the theoretical framework of the positive activity model (Lyubomirsky \& Layous, 2013) and previous research (Schueller, 2010), task enjoyment and difficulty were selected to investigate further. We aimed to lead the way in informing the positive activity model about the role of fit in smartphone-based intervention delivery. It was anticipated that the user's experience would relate to intervention outcome; specifically, it was hypothesized that a significant positive correlation would be observed between task enjoyment and wellbeing gain, and a significant positive correlation would be observed between task ease and wellbeing gain.

\section{Method}

\section{Participants}

As a fundamental aim of this study was to attract a self-selected pool of happiness seekers, participants were recruited through advertisements in two self-development based enewsletters and interest groups of this nature on Facebook and LinkedIn over a six weeks period. All participants signed up to the study "aimed at enhancing wellbeing" on a voluntary basis and were not offered any kind of incentives in return for taking part. Advertisments stipulated eligible participants needed to be over 18 years of age and have daily access to a smartphone. A total of 535 people enrolled on the study website, however, 341 people only partially completed stage 1 so their data was disqualified. Of the remaining 194 completing stage one, attrition reduced the number completing the second stage to 121 people (See Figure 1 for a flow diagram). The final sample of 121 participants $(86.6 \%$ female) represented eleven countries (including Australia, USA, Poland, Switzerland, Malta, Sweden, and Singapore). Ethnicity of the pool was predominantly Caucasian (90.1\%), compared with Asian/Pacific Islander (1.7\%), Hispanic (1.7\%), other/multiracial (5.0\%) and 1.5\% who declined to respond. Only $6.6 \%$ of the population were students. Responses indicated the pool was predominantly 
employed (65.3\%), postgraduate-level educated (41.3\%), married (51.2\%), with an annual income of $\$ 50,000$ - $\$ 74,999$ AUD (21\%). The mean age was 40.7 years $(S D=10.6)$.

All baseline questionnaires and the instructions explaining how to download the appropriate application to a smartphone device were stated on the study website. The website was accessible via smartphone, tablet or computer meaning participants could use their preferred device to go online and sign up. Email reminders were sent out to prompt participants to return to the website and complete follow up measures. Therefore, there was no face-to-face interaction between the experimenter and participants throughout the entire study.

Figure 1. CONSORT Flow Diagram

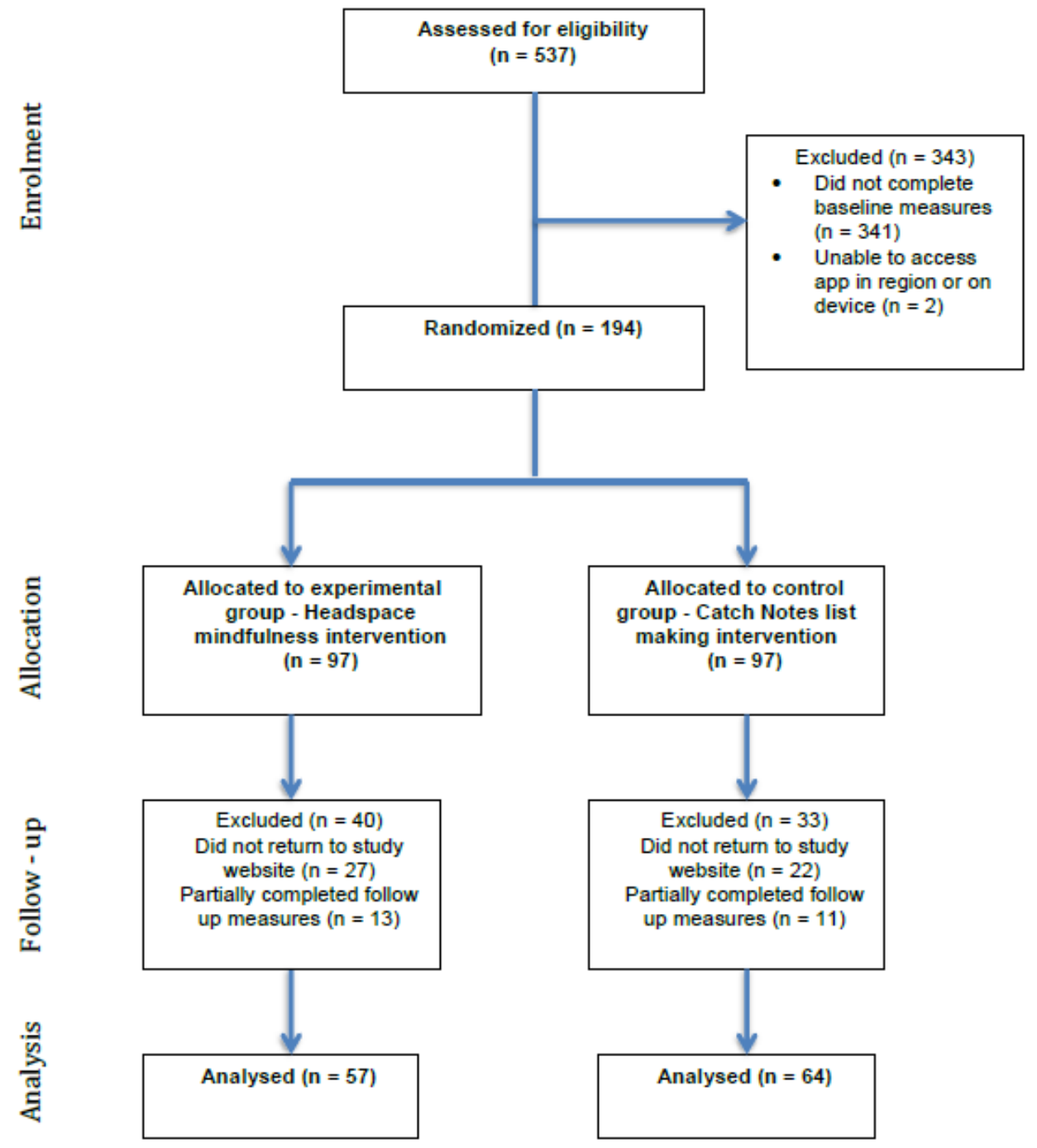




\section{Procedure}

Participants followed online study advertisements to a Survey Gizmo web page introducing the experiment and obtaining consent. Those taking part were advised that participation was voluntary and they had the right to withdraw at any time (at which point their data would be destroyed). Demographic information was gathered before a battery of baseline psychological measures were completed. After this, study instructions were disseminated according to the participant's randomly assigned condition. Although adverts presented this experiment as one aimed at enhancing wellbeing; website software randomly allocated participants to an experimental or control condition and participants remained unaware of this. A different smartphone application was selected to provide intervention content to each condition over 10 days.

\section{Headspace (Experimental Condition Application):}

The experimental condition $(n=57)$ engaged with an empirically based intervention. Headspace On-The-Go is a smartphone application delivering simple daily activities based on mindfulness practice. It teaches beginners the basic concepts of mindfulness through simple guided meditations and content is supported by science. Participants were instructed to follow the daily mindfulness exercises feature of the "Take 10" program for 10 minutes a day over 10 days. The application was brief and easy to use, free to download and accessible to smartphones globally.

\section{Catch Notes (Control Condition Application):}

The control condition $(n=64)$ engaged with a neutral task; a list-making application called Catch Notes. Although Catch Notes has a variety of organizational features, participants were instructed to use only the checklist function to "create an outline of what they did on this day last week" for 10 minutes a day over 10 days, and to "write out these activities in a checklist format" (adapted from Lyubomirsky et al., 2011). Catch notes was free to download, simple to 
use and available globally.

Screen-shot pictures were included on the study website to explain relevant application features and guide participants through the process of downloading the application and completing the assigned exercises. An optional two-minute promotional video was also offered in each condition for participants to familiarize themselves with application features. Participants were encouraged to commence the first smartphone-based exercise immediately, and self-administering the intervention, integrate it in to their daily lives for 10 days. On the 11th day, a follow-up email was sent out inviting participants to return to the study website. Here they were asked to rate their intervention experience before completing matching follow up measures to those completed at baseline. After this, participants were thanked for volunteering their time, and fully debriefed about the true aims of the experiment in accordance with ethical guidelines.

\section{Measures.}

The study used a range of assessments with established reliability and validity to measure dimensions of wellbeing at baseline and follow up. A selection of questions was also presented post-intervention to capture subjective ratings of the intervention experience.

\section{Satisfaction with Life Scale}

The Satisfaction with Life Scale (SWLS, Diener, Emmons, Larson \& Griffin, 1985) is a 5 item self-report scale assessing respondents satisfaction with life (e.g. "In most ways my life is close to my ideal") rated on a 7-point Likert scale (1=strongly disagree, $7=$ strongly agree). The summation of scores results in a higher score indicating greater life satisfaction. It is a validated measure with high test-retest reliability $(r=.82)$ and also demonstrates high internal consistency $(\alpha=.87$; Diener et al., 1985). 


\section{Flourishing Scale}

The Flourishing scale (Diener, Wirtz, Tov, Kim-Prieto, Choi, Oishi \& Biswas-Diener, 2009) comprising of 8 statements, was created to measure social-psychological prosperity and is mostly used to complement prevailing measures of subjective wellbeing. The scale includes items such as "I lead a purposeful and meaningful life" and "My social relationships are supportive and rewarding" incorporating essential components of a number of contemporary wellbeing theories to assess major aspects of social-psychological functioning from the respondent's viewpoint. Items are rated on a 7 -point Likert scale $(1=$ strongly disagree to $7=$ strongly agree). Scores range from 8 (lowest possible) to 56 (highest possible). A higher score indicates a person possessing many psychological strengths and resources.

\section{Positive and Negative Affect Scale}

Positive and Negative Affect Scale (PANAS, Watson, Clark, \& Tellegen, 1988) contains a total of 20 positive and negative items for respondents to subjectively rate. Using a 5-point Likert scale $(1=$ very slightly or not at all to $5=$ extremely $)$, respondents rate the extent to which they experienced feelings and emotions over the previous week (e.g. "Excited" and "Guilty"). A sum of the ratings produces two scores; one calculating positive affect (PA) and one for negative affect (NA). This measure is widely used in psychological research given its high test-retest reliability and levels of internal consistency $(\alpha=.89$ for PA, $\alpha=.85$ for NA; Crawford \& Henry, 2004).

\section{Center for Epidemiologic Studies Depression Scale}

The Center for Epidemiologic Studies Depression Scale (CES-D; Radloff 1977) is a 20-item measure used to assess the existence and duration of depressive symptoms during the previous week. Ratings of each symptom range from "Rarely or none of the time (less than 1 day)" to "Most of all of the time (5-7 days)". Items include "I felt that people dislike me" and 
"I thought my life had been a failure" where a high scores indicates more symptoms. The CES$\mathrm{D}$ is one of the most widely used depression instruments (Shafer, 2006).

\section{Follow-up Assessment}

Participants in all conditions received an email reminder within 24 hours of completing the intervention directing them to the study website to complete a final batch of measures. If no action was taken, a maximum of three reminder emails were sent within five days of completion. At follow up, all participants were asked to indicate the number of days the activity was completed (from 1-10), and respond to two activity rating questions ("1. To what extent did you find completing the activity enjoyable?" ( $1=$ not at all $-7=$ extremely); "2. To what extent did you find the activity a difficult one to complete?" (1= very difficult $-7=$ very easy) as well as all validated measures explained above.

\section{Statistical analyses}

Baseline characteristics, retention and per protocol results were compared among groups using bivariate statistics ( $\mathrm{T}$ and Chi squared tests). Bivariate comparisons were also carried out with retention as dependent variable and study group as independent variable controlling for socio-demographic and clinical characteristics. As there was only one follow up just a Per Protocol approach to analysis was used (Intent to Treat can be used if scores can be inferred from middle assessments). In addition to analysis of efficacy, Intention to Treat and Per Protocol analyses were carried out using repeated measures analysis of variance with SWLS, Flourishing, PANAS and CESD scores as dependent variables. Correlational analyses were conducted to explore whether gains in wellbeing experienced by the experimental group were related to participant's subjective ratings of task enjoyment, and task difficulty. 


\section{Results}

As can be seen in table 1, baseline characteristics (both socio-demographic characteristics and baseline outcome scores) were equivalent at the beginning of the trial. Only the level of negative emotions (measured with the PANAS scale) was found to statistically predict drop out from the study with a higher level for participants dropping out $(\mathrm{t}=-2.218$, $\mathrm{p}=.028)$.

\subsection{Intervention Efficacy}

Efficacy results using a per-protocol approach to analysis can be seen in table 2 . Statistically significant improvements were found for positive affect (measured with the PANAS scale) and depression (measured with the CESD scale). Results measured with PANAS are more robust in terms of effect size and power.

Table 1. Baseline Socio-demographic and wellbeing Characteristics by experimental group

\begin{tabular}{|c|c|c|c|c|c|}
\hline & Experimental & $(n=97)$ & Control & $(n=97)$ & \\
\hline \multicolumn{6}{|l|}{$\begin{array}{l}\text { SOCIO- } \\
\text { DEMOGRAPHICS }\end{array}$} \\
\hline & $\mathrm{N}$ & $\%$ & $\mathrm{~N}$ & $\%$ & Significance \\
\hline Gender ( $\%$ female $)$ & 83 & 85.6 & 88 & 90.7 & $\begin{array}{l}\chi^{2}=1.223 \\
p=.267\end{array}$ \\
\hline Employment (Active, \%)* & 91 & 93.8 & 91 & 93.8 & $\begin{array}{l}\chi^{2}=.000 \\
p=1.000\end{array}$ \\
\hline Couple status $* *$ (married \%) & 50 & 51.5 & 47 & 48.5 & $\chi^{2}=186, p=.667$ \\
\hline Single & 34 & 35.1 & 37 & 38.1 & \\
\hline Divorced/separated & 13 & 13.3 & 13 & 13.3 & \\
\hline $\begin{array}{ll}\text { Education } & \text { (University } \\
\text { degree } \%) & \end{array}$ & 76 & 78.4 & 68 & 70.1 & $\begin{array}{l}\chi^{2}=1.724 \\
\mathrm{p}=.189\end{array}$ \\
\hline & $\mathrm{M}$ & SD & $\mathrm{M}$ & SD & \\
\hline Age & 39.74 & 10.84 & 40.86 & 10.27 & $\mathrm{t}=-.734, \mathrm{p}=.464$ \\
\hline \multicolumn{6}{|l|}{ WELLBEING } \\
\hline SWL & 22.56 & 6.74 & 22.04 & 7.23 & $\mathrm{t}=.513 \cdot \mathrm{p}=.608$ \\
\hline Flourishing & 44.68 & 7.90 & 43.90 & 7.89 & $\mathrm{t}=.691 . \mathrm{p}=.490$ \\
\hline Positive affect & 32.43 & 7.78 & 33.27 & 9.35 & $\mathrm{t}=-.676 . \mathrm{p}=.500$ \\
\hline Negative affect & 19.10 & 7.39 & 19.99 & 8.40 & $\mathrm{t}=-.780 . \mathrm{p}=.436$ \\
\hline Depression (CESD) & 15.54 & 12.40 & 15.30 & 12.37 & $\mathrm{t}=-.133 . \mathrm{p}=.894$ \\
\hline
\end{tabular}

*Includes all people working, homemaking or studying vs. unemployed or unable to work. **The variable was dichotomized (with couple vs. rest) in order to make subsamples statistically comparable due to low frequencies in the widow category.

SWL: Satisfaction with Life Scale

CESD: Center for Epidemiologic Studies Depression Scale 
Cite as: Howells, A., Ivtzan, I., \& Eiroa-Orosa, F. J. (2016). Putting the "app" in Happiness: A Randomised Controlled Trial of a Smartphone-Based Mindfulness Intervention to Enhance Wellbeing. Journal of Happiness Studies, 17(1), 163-185. https://doi.org/10.1007/s10902-014-9589-1

Table 2. Follow up and baseline wellbeing scores by experimental group and results of the repeated measures analyses of variance

\begin{tabular}{|c|c|c|c|c|c|c|c|c|c|c|c|}
\hline & \multicolumn{4}{|c|}{ Experimental $(n=57)$} & \multicolumn{7}{|c|}{ Control $(n=64)$} \\
\hline & Pre & & Post & & Pre & & Post & & & & \\
\hline & $\mathrm{M}$ & $\mathrm{SD}$ & $\mathrm{M}$ & $\mathrm{SD}$ & $\mathrm{M}$ & $\mathrm{SD}$ & $\mathrm{M}$ & $\mathrm{SD}$ & Significance & $\begin{array}{l}\text { Effect } \\
\text { Size }\left(\eta_{\mathrm{p}}{ }^{2}\right)\end{array}$ & Power \\
\hline SWL & 23.09 & 6.29 & 24.61 & 5.91 & 21.95 & 6.26 & 22.91 & 5.95 & $\mathrm{~F}=.396, \mathrm{p}=.530$ & .003 & .096 \\
\hline Flourishing & 45.49 & 6.80 & 47.68 & 5.18 & 43.89 & 6.92 & 45.31 & 7.48 & $\mathrm{~F}=.746, \mathrm{p}=.389$ & .006 & .137 \\
\hline Positive affect & 31.96 & 7.74 & 34.47 & 7.41 & 33.53 & 9.28 & 31.88 & 8.47 & $\mathrm{~F}=9.133, \mathrm{p}=.003$ & .071 & .850 \\
\hline Negative affect & 18.40 & 7.50 & 16.00 & 5.55 & 18.73 & 6.66 & 17.72 & 5.86 & $\mathrm{~F}=1.246, \mathrm{p}=.389$ & .010 & .198 \\
\hline Depression & 14.09 & 11.58 & 10.05 & 9.13 & 14.31 & 10.97 & 13.39 & 9.59 & $\mathrm{~F}=3.723, \mathrm{p}=.05$ & .030 & .482 \\
\hline
\end{tabular}




\subsection{Wellbeing and person-activity fit}

As PANAS positive affect showed the most robust results, we decided to carry correlational analyses with task enjoyment and difficulty. A statistically significant correlation between positive affect gains and task enjoyment $(r=.285, p=.031)$, but no correlation with task difficulty rating $(r=0.095, \mathrm{p}=.482)$ were found. In comparison, for the control group, any gains in positive affect were not significantly correlated with task difficulty $(r=-.115, \mathrm{p} .367)$ or task enjoyment $(r=-.083, p=.512)$.

\section{Discussion}

A number of important findings were uncovered within the present research; relating to positive intervention delivery, methodology and experience. In the first randomized controlled positive intervention study via smartphone, happiness seekers were found to significantly benefit from a mindfulness-based intervention. These findings offer new insight that enriches current understanding of the interplay between person, activity, method of delivery and wellbeing enhancement.

Results confirmed the first hypothesis by demonstrating the importance of an empirical basis to intervention content in order to significantly enhance wellbeing. Simultaneously, this hypothesis confirmed that it was possible to successfully deliver a mindfulness-based positive intervention via smartphone application. Finally, an exploration of person-activity fit showed a significant positive correlation between wellbeing gain and task enjoyment.

\section{Be Mindful that Content Counts}

This study used a sample of happiness seekers with a will to become happier, who believed they were all engaging with a positive intervention via smartphone application. Random allocation to an experimental or control condition established that empirically based content was crucial to improving wellbeing. Results showed that 
engagement with the mindfulness application lead to significant gains in positive affect with a medium effect size and reduced depressive symptoms with a small effect size. No significant gains were observed in the control condition. The combination of happiness seeker's motivation and a placebo task revealed that self-selection was not enough to markedly enhance one's wellbeing. As hypothesised, the combination of empirical content and a happiness-seeking attitude was. The mindfulness-based application content was an effective choice of intervention as it yielded a significant positive impact on wellbeing in ten days or less. This is comparable to other forms of brief mindfulness training (Cavanagh et al., 2013; Harnett et al., 2010). Although meditation has previously been identified as the least practiced intervention strategy by those purposefully pursuing happiness (Parks et al., 2012), such promising results suggest mindfulness-based interventions deserve more attention in the positive psychological intervention literature and by those seeking to enhance their wellbeing levels.

The results of this investigation underscore the importance of engaging with content based on scientific understanding if one's goal is to become significantly happier. The findings support earlier outcomes such as Lyubomirsky et al. (2011) demonstrating that beyond self-selection; interventions need scientific substance. It is optimal to have both the intention to become happier, as well as the right intervention content, for benefits to transpire and last. These results evidence that empirically based interventions, when used in real-world settings, can be highly beneficial to those motivated to use them. Thus the development and application of accessible positive interventions is an important undertaking for the future of this field. The translation of empirical findings into interventions that are widely disseminated and readily available to those they were intended for, could afford happiness seekers the greatest benefits 
from their pursuit. Furthermore, this objective supports the central mission of the founder of positive psychology - to markedly enhance human flourishing worldwide in the twenty-first century (Seligman, 2011).

\section{The Smarter Approach}

As other fields have successfully adopted smartphone-based methodologies, the viability of a positive intervention delivered via smartphone application was explored within this investigation. Only one study to date in the field of positive psychology has analysed the efficacy of a smartphone-based suite of interventions (Parks et al., 2012). However, this study did not use random assignment or a control condition, meaning the efficacy of the intervention could be disputed, as it was impossible to draw certain conclusions from these findings.

Layous and Lyubomirsky (2014), Bolier et al. (2013), Mazzuchelli et al. (2012) and Sin and Lyubomirsky (2009) present numerous randomized controlled experiments successfully demonstrating how intentional changes to thoughts and behaviours create meaningful increases in wellbeing. The gamut of positive interventions has been previously delivered in a variety of formats including face-to-face, web-based, telephone and books. Yet format of delivery has been identified as a potential moderator impacting upon intervention effectiveness (Lyubomirsky \& Layous, 2013; Nelson \& Lyubomirsky, 2012). This study has extended existing research by Parks et al., (2012) exploring the viability of smartphones to deliver interventions with randomised controlled trial results showing that this format is comparable to existing methods used to successfully enhance wellbeing.

Miller (2012) claims the formidability of smartphones is enough to "transform psychology even more profoundly than PCs and brain imaging" (p.221). By only using basic smartphone features through a downloadable application, the current findings 
suggest that smartphones are a feasible tool by which to disseminate intervention content successfully; extending contemporary web-based delivery (e.g. Schueller \& Parks, 2012; Mitchell, Vella-Brodrick \& Klein, 2010; Schueller, 2010) to an even more accessible, cost-effective and user-friendly platform. The versatility of these devices means their functionality could be used in many ways to benefit both the study and enhancement of the human experience. It is hoped that this maiden trial inspires other researchers to follow suit and push methodological boundaries.

\section{If the Intervention Fits}

This study explored the relationship between person and activity features, hypothesising a significant positive relationship would be observed between gains in wellbeing and the degree of person-activity fit (derived from task enjoyment and task difficulty ratings). The hypotheses driving this exploration were guided by the positive activity model (Lyubomirsky \& Layous, 2013). Analysis of the user experience showed in the case of those engaging with a validated positive intervention; wellbeing gains were related to task enjoyment.

The obtained results show a strong relationship exists between how much a person relishes the experience of the intervention and their eventual wellbeing gain. Previous literature suggests that happiness seekers do not always know best, and rather than pursue optimal strategies that "fit", they may instead engage with interventions on the basis of enjoyment, or ease, that are not necessarily the most effective for them (Parks et al., 2012; Study 3). In considering the vast choice of self-help applications available, users are likely to engage with an intervention that has these qualities, however, this is not indicative of the intervention working. The results obtained in this investigation found no correlation in the control condition between wellbeing, task ease or enjoyment rating. It appears logical that people who enjoyed the task, and found it 
easy would make greater wellbeing gains (as opposed to not enjoying it and finding it challenging), supporting previous research on the importance of person-activity fit and user preference (Schueller, 2010, 2011). It would be easy to speculate that people expressed enjoyment in the task because their wellbeing levels were increasing, however, correlation does not imply causation and this directional assumption requires additional research. Further, the observed relationships may have been moderated by a third factor, such as the level of effort exerted, or the kind of intervention delivered, which also demonstrates the need for future research exploring fit and gains made in this domain.

Whilst these results have uncovered interesting findings regarding fit and smartphone delivery, it is important to qualify that these results cannot be generalized to all positive interventions delivered in this way. The present study used a mindfulnessbased intervention, employing its own approach to wellbeing enhancement using a different combination of techniques to that of writing a letter of gratitude (e.g. Layous, Lee, Choi, \& Lyubomirsky, 2013); finding new ways to use character strengths (Seligman et al., 2005); practicing optimism (e.g. Layous et al., 2012) and so on. There are still significant gaps in knowledge between laboratory and real world settings, with results that could have important repercussions for those authentically pursuing happiness and also the future delivery platforms for interventions. It cannot be assumed that the most effective and prominent interventions will be as effective across all formats of delivery, inversely, it may be that the lesser utilized and unfamiliar exercises will gain notoriety via smartphone application instead. It is also likely that fit will be conceptualized, measured and emphasised differently across intervention platforms in time. With thousands of contending smartphone applications, it is anticipated that a dynamic approach to intervention delivery based on user feedback of fit levels will be 
necessary to encourage self-administration and ongoing engagement if significant positive outcomes are to be attained.

\section{Caveats and Limitations}

\section{Real-World Approach.}

This study attempted to research behaviour as it is likely to occur in a real world context. In doing so, the internal validity is somewhat compromised by the heightened ecological validity. It is believed to be a worthwhile trade off in order to better understand phenomena in the context that it naturally happens and by designing a randomized control trial it was anticipated that these effects would be minimized, with a decent level of internal validity remaining. However, the lack of internal control remains noteworthy.

\section{Sample.}

Every effort was made to attract a global sample of active happiness seekers. Although a diverse sample was collected, it is acknowledged that there are still limitations to the generalizability of findings to happiness seekers from all cultures. The sample was predominantly well-educated Caucasian females living in Australia, with an average age of 40.7 years. It is also acknowledged that not every happiness seeker owns, or engages with smartphone technology or applications and this may have created biases in the chosen sample by representing a specific subgroup within this target population. In defence of the present study, demographic information revealed that participants ranged in age from 19 to 63 years and were located across eleven countries, suggesting far greater diversity than most homogenous student samples used in psychological research. Further, a population of users circa 1.82 billion (Khalaf, 2013) challenges the notion that this prerequisite was restrictive to participation. 
The sample used in the present study was a self-selecting population. Lyubomirsky et al. (2011) reason that respondents in a self-selecting sample will be fully aware of the activities they are engaging in (e.g. Seligman et al., 2005), and may therefore express biases that result in higher reported wellbeing gain. If that was the case in the present study, we should have seen convergence in the reported wellbeing gain between the control and experimental groups. Instead, we observed the control group reporting a slight wellbeing loss, while the experimental group reported significant gain in positive affect and significant reduction in depressive symptoms. This suggests no impact of expectation of wellbeing gain. Although the population was self-selected, the presumption of gaining and the influence of bias upon wellbeing gain was weak.

\section{Attrition.}

The study advertisments attracted 535 people to follow links and enrol on the study website. This number reduced dramatically to 194 participants fully completing stage one indicating an attrition rate of $63.74 \%$. A further participant attrition rate of $37.95 \%$ between baseline and follow up reduced the final number of participants to 121. This was higher than anticipated and indicates potentially higher rates for smartphone-based research compared to other methodologies. It is plausible that this reduction in numbers may have resulted from the voluntary nature of participation, the lack of face-to-face interaction, or perhaps reflected methodological limitations including participant distraction, loss of interest, a lack of compatibility between the individual's device and website content, low battery or poor internet signal. These and other similar issues are to be expected when selecting this methodology (for a review, see Miller, 2012), although it is anticipated that novel ways to overcome them will evolve over time as smartphone research matures. 


\section{Applications.}

This investigation used existing smartphone applications, as resource constraints did not permit the design and creation of custom-built ones. The selected applications met intervention criteria, were free to download and available globally. However, the available content also determined the duration of the intervention and restricted delivery to ten days. When adopting smartphone-based methodology, it would be ideal to collect and access data via a purpose-built customised application. This approach would allow for smoother integration of the user experience, give the experimenter greater control over content and duration, as well as provide a richer dataset including analytics on duration and frequency of usage, time of day, functions used and much more (Parks et al., 2012). Although cost, programming and analysis obstacles prohibited this approach from being adopted in the present study; it is noteworthy that a collaborative development may have further enriched this investigation and afforded greater flexibility in terms of intervention content, dissemination and exposure.

\section{To add in?}

Although no significant differences were observed in satisfaction with life or flourishing scores, it is possible that a longer exposure to the intervention may have lead to more favourable outcomes across these measures also. .... ALSO - No follow up the study may have needed a longer follow-up period to determine long-term gains in wellbeing across the multiple measures used.

\section{Future Directions}

\section{Think Smarter.}


The findings of this investigatio in support the idea that interventions can be successfully delivered within application format. Moderate and small effect sizes were captured, making outcomes from a smartphone-based intervention comparable to other forms of intervention (Bolier et al., 2013; Sin \& Lyubomirsky, 2009). Meta-analysis has previously identified self-administered interventions as the least effective delivery format, whereas human-supported interventions yield larger effect sizes (Sin \& Lyubomirsky, 2009). Therefore, it would be valuable for future research to explore the comparison or addition of smartphone-based application delivery to alternative formats. It may be the case that smartphone-based interventions are even more effective when supplemented by other means of intervention delivery, for example, adjunct to a human-supported intervention such as coaching. One anticipated benefit of combining smartphone and face-to-face intervention delivery would be increased adherence to an approach that so easily permits high user turnover.

Smartphone technology is underutilized in psychological research at large and the scalability potential of intervention delivery is yet to be fully recognized. This investigation marks an important step in rigorous research to uncover the costs and benefits of integrating wellbeing investment and positive interventions in the real world using feasible methods. The global interest in attaining happiness (Diener, 2000; Goldberg et al., 2009) continues to maintain widespread appeal - affirmed by the number of happiness seeking volunteers this study attracted. Such popularity coupled with unprecedented smartphone usage and rising reliance on applications (Khalaf, 2013), strongly suggests that smartphone-based methodology and engagement via application is a rational progression for wellbeing researchers to make.

An opening has been created whereby digital access could diminish the geographical, cultural, and financial barriers to individuals accessing positive 
interventions, whilst researchers benefit from studying life as it is lived. The functionality of smartphone devices can be used to enhance existing interventions in novel ways; for example capturing a video of a random act of kindness, recording geolocations where wellbeing is enhanced or tracking the social contagion of happiness. Herein lies a tangible opportunity for positive psychology and happiness seekers to apply the science of human flourishing in an innovative, meaningful and comprehensive way (Seligman, 2011).

It is essential for psychology research to explore twenty-first century practices and relate scientific understanding to present-day experience, as current trends are valuable indicators of future research opportunities. For example, recent market research revealed that smartphone users spend more time in applications than online, and interactive gaming (accounting for $43 \%$ of all usage in America) is the most popular activity (Khalaf, 2013). Gamification, where elements of game playing are applied to tasks, can be used to engage, educate and enhance personal resources in interactive ways. Early research on gaming has provided favourable results concerning intervention engagement and behaviour change across a range of health contexts (Mohr et al., 2013). Applications such as Happify (2012) and Superbetter (2012) that integrate gaming and positive psychology principles, showcase how dynamic the ways to wellbeing can be. Creative collaborations of this nature and beyond will prove fruitful in future understanding of intervention delivery, user engagement and optimizing positive behavioural outcomes with the use of technology.

\section{Theoretically speaking.}

As a ten-day intervention study, this investigation offers an insightful snapshot of human experience whilst serving as a valuable primer for longitudinal research. It is 
anticipated that this investigation will contribute towards addressing the theoretical gap surrounding smartphone-based interventions, and future research will further extend these findings to reveal the sustainability and long-term effects of the smartphone-based approach on wellbeing. There are multiple factors of positive interventions and those engaging in them that were beyond the scope of the present study; such as personality, optimal dosage and duration of intervention. Future longitudinal research will give a clearer insight in to the impact of such factors on wellbeing investment and how this format of delivery fares for a diverse and global user population over time.

Given the unique interaction between user and device, it is not sufficient to simply revise existing behaviour change models intended for other platforms to support the design and analysis of interventions delivered via smartphone. For example, while Ritterband et al. (2009) have developed a model of behaviour change for internet-based interventions, one cannot assume this will directly translate to the distinctive experience of smartphone users engaging with an application. Mobile and internet-based interventions are in some ways comparable, but are certainly not the same. Acknowledging this distinction, Riley et al. (2013) argue that mobile interventions require their own guiding theory that captures their dynamic nature, and that which sets them apart from other forms of intervention. Interestingly, Webb (2010) found the effect size of internet-based interventions increased in relation to their theoretical basis. Given the limited attention smartphone research has received thus far, it is not yet known whether the same is true for interventions of this nature.

\section{Applying Positive Psycholog.y}

The impressive evolution of positive psychological interventions reflects the flourishing state of wellbeing research at a time when there is widespread need. Research and application are progressing almost simultaneously and this synergy is 
expected to continue. For example, the meta-analyses by Sin and Lyubomirsky, (2009) advocated a varied "shotgun" approach to interventions is most effective; in which individuals practice multiple strategies to boost wellbeing at the same time. Shortly thereafter, the Livehappy application was created, offering users eight exercises to boost wellbeing focusing on domains such as gratitude, savouring, and acts of kindness (Parks et al., 2012, Study 3). This investigation demonstrated the relative ease with which the science can be disseminated to its supporters. As more is discovered regarding the optimal conditions for wellbeing enhancement, it is anticipated this will be rapidly translated in to new and dynamic forms of intervention that offer truly accessible ways to wellbeing.

A prospective focus for the future concerns regulating and testing self-help content by means of applying scientific rigour. So-called wellbeing enhancing content requires a form of governing such as a seal of approval by experts if it is deemed efficacious. Specifically in the field of positive psychology, clear distinctions must be made between effective wellbeing interventions and non-empirical or harmful self-help alternatives, for the sake of the user and also to maintain the credibility of this field (Norrish \& Vella-Brodrick, 2007; see also Ehrenreich, 2010; Lazarus, 2003).

\section{Conclusion}

The founder of the field of positive psychology ambitiously declared that by $2051,51 \%$ of the world's population will be flourishing (Seligman, 2011). It is widely accepted that positive interventions are a feasible and sustainable method of facilitating 
human flourishing through markedly increasing wellbeing; thus offering a way of moving towards this goal. Seligman (2011) accentuated the crucial role of positive computing in rising to the colossal challenge, as well as the contagion of happiness (Fowler \& Christakis, 2008). The consequent rise of the smartphone adds an incredibly important dimension to this operation, and brings with it a network of over 1.82 billion users (Khalaf, 2013); one quarter of the current population of the world. With this in mind, the present study has covered new territory to demonstrate that smartphones are a viable, effective method of intervention dissemination that offer a way to make happiness seekers significantly happier. Whilst much more work needs to be done, the present study presents a case for smartphone-based interventions to support the inspirational vision of this field.

\section{Acknowledgements}

The authors would like to thank colleagues, friends and family for their valuable support, guidance and feedback throughout the project. Special thanks also to Dr Timothy Sharp at The Happiness Institute and Rachel Green at The Emotional Intelligence Institute for their support in recruiting participants, to Headspace for agreed usage of their smartphone application and to all the Happiness Seekers who volunteered their time and energy to take part. 


\section{References}

Baer, R. A. (2003). Mindfulness training as a clinical intervention: A conceptual and empirical review. Clinical Psychology: Science and Practice, 10, 125-143.

Baer, R. A.,. Lykins, E. L. B., \& Peters, J. R. (2012). Mindfulness and selfcompassion as predictors of psychological wellbeing in long-term meditators and matched nonmeditators. Journal of Positive Psychology, 7(3), 230238.

Boehm, J. K., Lyubomirsky, S., \&Sheldon, K. M. (2011a). A longitudinal experimental study comparing the effectiveness of happiness-enhancing $\mathrm{s}$ trategies in Anglo Americans and Asian Americans. Cognition and Emotion, $25,1152-1167$.

Bolier, L., Haverman, M., Westerhof, G. J., Riper, H., Smit, F., \& Bohlmeijer, E. (2013). Positive psychology interventions: a meta-analysis of randomized controlled studies. BMC Public Health, 13:119.

Boulos, M. N. K., Wheeler, S., Tavares, C. \& Jones, R. (2011). How smartphones are changing the face of mobile and participatory healthcare: an overview, with example from eCAALYX. BioMedical Engineering OnLine, 10:24.

Brown, K. W., Ryan, R. M., \& Creswell, J. D. (2007). Mindfulness: Theoretical Foundations and Evidence for its Salutary Effects. Psychological Inquiry, 18, 211-237.

Busis, N. (2010). Mobile phones to improve the practice of neurology. Neurologic Clinics, 28(2), 395-410.

Carmody, J., \& Baer, R. A. (2009). How long does a mindfulness-based stress reduction program need to be? A review of class contact hours and effect sizes for psychological distress. Journal of Clinical Psychology, 65(6), 627-638.

Cavanagh, K., Strauss, C., Cicconi, F., Griffiths, N., Wyper, A., \& Jones, F., (2013). A randomised controlled trial of a brief online mindfulness-based intervention. Behaviour Research and Therapy, 51(9), 573-578.

Clough, B. A., \& Casey, L. M., (2011). Technological adjuncts to enhance current psychotherapy practices: A review. Clinical Psychology Review, 31(3), 279292.

Cohn, M.A., \& Fredrickson, B.L. (2010). In search of durable positive psychology interventions: Predictors and consequences of long-term positive behavior 
change. The Journal of Positive Psychology, 5, 355-366.

Crawford, J. R., \& Henry, J. D. (2004). The Positive and Negative Affect Schedule (PANAS): Construct validity, measurement properties and normative data in a large non-clinical sample. British Journal of Clinical Psychology, 43, 245- 265.

Diener, E. (2000). Subjective well-being: The science of happiness and a proposal for a national index. American Psychologist, 55, 34-43.

Diener, E., Emmons, R. A., Larsen, R. J., \& Griffin, S. (1985). The Satisfaction With Life Scale. Journal of Personality Assessment, 49, 71-75.

Diener, E., Wirtz, D., Tov, W., Kim-Prieto, C., Choi, D., Oishi, S., \& Biswas-Diener, R. (2009). New measures of well-being: Flourishing and positive and negative feelings. Social Indicators Research, 39, 247-266.

Dillbeck, M. C., \& Orme-Johnson, D. W. (1987). Physiological differences between transcendental meditation and rest. American Psychologist, 42(9), 879-881.

Dufau, S., Dunabeitia, J. A., Moret-Tatay, C., McGonigal, A., Peeters, D., Alario, X., . . Grainger, J. (2011). Smart phone, smart science: How the use of smartphones can revolutionize research in cognitive science. PLoS ONE, 6, e24974.

Dulin, P. L., Gonzalez, V. M., King, D. K., Giroux, D. \& Bacon, S. (2013). Development of a Smartphone-Based, Self-Administered Intervention System for Alcohol Use Disorders. Alcoholism Treatment Quarterly, 31(3), $321-336$.

Emmons, R.A., \& McCullough, M.E. (2003). Counting blessings versus burdens: An experimental investigation of gratitude and subjective wellbeing in daily life. Journal of Personality and Social Psychology, 54, 466-475.

Ferguson, Y. L., \& Sheldon, K. M. (2013). Trying to be happier really can work: Two experimental studies. The Journal of Positive Psychology, 8(1), 23-33.

Fowler, J. H., \& Christakis, N. A. (2008). The Dynamic Spread of Happiness in a Large Social Network: Longitudinal Analysis Over 20 Years in the Framingham Heart Study, British Medical Journal, 337: a2338.

Geschwind N., Peeters F., Drukker M., Van Os J., Wichers M. (2011). Mindfulness t raining increases momentary positive emotions and reward experience in adults vulnerable to depression: a randomized controlled trial. Journal of Consulting Clinical Psychology, 79, 618-628. 
Glück, T. M., \& Maercker, A. (2011). A randomized controlled pilot study of a brief web-based mindfulness training. BMC Psychiatry, 11:175.

Goldberg, A. B., Fillmore, N., Andrzejewski, D., Xu, Z., Gibson, B., \& Zhu, X. (2009). May all your wishes come true: A study of wishes and how to recognize them. In Human language technologies (pp. 263-271). Boulder, CO: Association for Computational Linguistics.

Haidt, J. (2006). The happiness hypothesis. New York, NY: Basic Books.

Harnett, P. H., Whittingham, K., Puhakka, E., Hodges, J., Spry, C., \& Dob, R. (2010). The short-term impact of a brief group-based mindfulness therapy program on depression and life satisfaction. Mindfulness, 1(3), 183-188.

Hart, R., Ivtzan, I., \& Hart, D. (2013). Mind the Gap in Mindfulness Research: A comparative account of the leading schools of thought. Review of General Psychology, 17(4), 453-466.

Hebden, L., Cook, A., Van der Ploeg, H. P., \& Allman-Farinelli, M. (2012).

Development of Smartphone Applications for Nutrition and Physical Activity Behavior Change. JMIR Research Protocols, 1(2), e9.

Intille, S. S. (2012). Emerging technology for studying daily life. In Handbook of research methods for studying daily life. Mehl, Matthias R. (Ed.); Conner, Tamlin S. (Ed.); pp. 267-282. New York, NY, US: Guilford Press.

Ivtzan, I., Gardner, H. E., \& Smailova, Z. (2011) Mindfulness meditation and curiosity: The contributing factors to wellbeing and the process of closing the selfdiscrepancy gap. International Journal of Wellbeing, 1(3), 316-326.

Jislin-Goldberg, T., Tanay, G., \& Bernstein, A. (2012). Mindfulness and positive affect: Cross sectional, prospective intervention, and real-time relations. The Journal of Positive Psychology, 7(5), 349-361.

Kabat-Zinn, J. (2003). Mindfulness-based interventions in context: Past, present, and future. Clinical Psychology: Science and Practice, 10, 144-156.

Kabat-Zinn, J. (2005). Coming to our senses: Healing ourselves and the world through mindfulness. New York, NY: Hyperion.

Keng, S., Smoski, J. J., \& Robins, C. J. (2011). Effects of mindfulness on psychological health: A review of empirical studies. Clinical Psychology Review, 6 (31), 
1041-1056.

Khalaf, (2013). Flurry Five-Year Report: It's an App World. The Web Just Lives in It.

Retrieved on August 21, 2013, from: http://blog.flurry.com/bid/95723/Flurry-

Five-Year-Report-It-s-an- App-World-The-Web-Just-Lives-in-It.

Killingsworth, M. A., \& Gilbert, D. T. (2010). A wandering mind is an unhappy mind.

Science, 330, 932.

Kunda, Z. (1990). The case for motivated reasoning. Psychological Bulletin, 108, 480498.

Layous, K., Lee, H. C., Choi, I, \& Lyubomirsky, S. (2013). Culture matters when designing a successful happiness-increasing activity: A comparison of the United States and

South Korea. Journal of Cross-Cultural Psychology, 44,1294-1303.

Layous, K., \& Lyubomirsky, S. (2014). The how, why what, when, and who of happiness: Mechanisms underlying the success of positive interventions. In $\mathrm{J}$. Gruber \& J.

Moscowitz (Eds.), Positive emotion: Integrating the light sides and dark sides (pp. 473-495). New York: Oxford University Press.

Layous, K., Nelson, S. K., \& Lyubomirsky, S. (2012). What is the optimal way to deliver a positive activity intervention? The case of writing about one's best possible selves. Journal of Happiness Studies, 14(2), 635-654.

Lazarus, R. (2003). Does the positive psychology movement have legs? Psychological Inquiry, 14(2), 93-109.

Luxton, D. D., McCann, R. A., Bush, N. E., Mishkind, M. C., \& Reger, G. M. (2011). mHealth for mental health: Integrating smartphone technology in behavioral healthcare. Professional Psychology: Research and Practice, 42(6), 505-512.

Ly, K. H., Dahl, J., Carlbring, P., \& Andersson, G. (2012). Development and initial evaluation of a smartphone application based on acceptance and commitment therapy. SpringerPlus, (1),1- 11 .

Lykken, D., \& Tellegen, A. (1996). Happiness is a stochastic phe- nomenon. Psychological

Science, 7, 186-189. 
Lyubomirsky, S., Dickerhoof, R., Boehm, J. K., \& Sheldon, K. M. (2011). Becoming happier takes both a will and a proper way: An experimental longitudinal intervention to boost well-being. Emotion, 11, 391-402.

Lyubomirsky, S., King, L., \& Diener, E. (2005). The benefits of frequent positive affect:

Does happiness lead to success? Psychological Bulletin, 131, 803-855.

Lyubomirsky, S., \& Layous, K. (2013). How do simple positive activities increase wellbeing? Current Directions in Psychological Science, 22(1), 57-62.

Lyubomirsky, S., Sheldon, K. M., \& Schkade, D. (2005). Pursuing Happiness: The Architecture of Sustainable Change. Review of General Psychology, 9, 111-131. Mazzucchelli, T. G., Kane, R. T., \& Rees, C. S. (2010). Behavioural activation interventions

for wellbeing: A meta-analysis. Journal of Positive Psychology, 5(2), 105-121. McTavish, F. M., Chih, M., Shah, D., \& Gustafson, D. H. (2012). How patients recovering

from alcoholism use a smartphone intervention. Journal of Dual Diagnosis, 8(4), 294-304.

Miller, G. (2012). The Smartphone Psychology Manifesto. Perspectives on Psychological Science, 7(3), 221-237.

Mitchell, J., Vella-Brodrick D., \& Klein, B. (2010). Positive psychology and the internet: A mental health opportunity Well-Being. Journal of Applied Psychology, $6(2), 30-41$.

Mohr, D.C., Burns, M, N., Schueller, S. M., Clarke, G., \& Klinkman, M. (2013). Special Section: Health Information Technology and Mental Health Services Research: A Path Forward. Behavioral Intervention Technologies: Evidence review and recommendations for future research in mental health. General Hospital Psychiatry, 35, 332-338.

Nelson, S. K., \& Lyubomirsky, S. (2012). Finding happiness: Tailoring positive activities for optimal well-being benefits. In M. Tugade, M. Shiota, \& L. Kirby (Eds.), Handbook of positive emotions. New York: Guilford.

Norrish, J. M., \& Vella-Brodrick, D. A. (2007). Is the Study of Happiness a Worthy Scientific Pursuit? Social Indicators Research, 3, 393-407.

Ozdalga, E., Ozdalga, A., \& Ahuja, N. (2012). The Smartphone in Medicine: A Review of Current and Potential Use Among Physicians and Students, Journal 
of Medical Internet Research, 14(5), 142-155.

Parks, A. C., \& Biswas-Diener, R. (2013). Positive Interventions: Past, present and future. In T. Kashdan \& J. Ciarrochi (Eds.), Mindfulness, acceptance, and positive psychology: The seven foundations of well-being (pp.140-165). Oakland, CA: Context.

Parks, A., Della Porta, M., Pierce, R. S., Zilca, R., \& Lyubomirsky, S. (2012). Pursuing happiness in everyday life: The characteristics and behaviors of online happiness seekers. Emotion, 12(6), 1222-1234.

Radloff, L. S. (1977). The CES-D scale: a self-report depression scale for research in the general population. Applied Psychological Measurement, 1,385-401.

Raento, M., Oulasvirta, A., \& Eagle, N. (2009). Smartphones: An emerging tool for social scientists. Sociological Methods \& Research, 37(3), 426-454.

Riley, W. T., Rivera, D. E, Atienza, A. A., Nilsen, W., Allison, S. M., \& Mermelstein, R. (2011). Health behavior models in the age of mobile interventions: are our theories up to the task? Translational Behavioral Medicine, 1(1), 53-71.

Ritterband, L. M., Thorndike, F. P, Cox, D. J., Kovatchev, B. P., \& Gonder-Frederick, L. A. (2009). A behavior change model for internet interventions. Annals of Behavioral Medicine, 38(1), 18-27.

Schueller, S.M. (2010). Preferences for positive psychology exercises. Journal of Positive Psychology, 5, 192-203.

Schueller, S. M. (2011). To each his own well-being bosting intervention: using preference to guide selection. The Journal of Positive Psychology, 6(4), 300313.

Schueller, S. M., \& Parks, A. C. (2012). Disseminating self-help: Positive psychology exercises in an online trial. Journal of Medical Internet Research, 14, e63.

Seligman, M. E. P. (2011). Flourish: A visionary new understanding of happiness and well-being. NY: Free Press.

Seligman, M. E. P., Steen, T. A., Park, N., \& Peterson, C. (2005). Positive psychology 
progress: Empirical validation of interventions. American Psychologist, 60, $410-421$.

Seligman, M. E. P., \& Csikszentmihalyi, M. (2000). Positive psychology: An introduction. American Psychologist, 55, 51-82.

Shafer, A. B. (2006). Meta-analysis of the factor structures of four depression questionnaires: Beck, CES-D, Hamilton, and Zung. Journal of Clinical Psychology, 62(1), 123-146

Shapiro, S. L., \& Carlson, L. E. (2009). The art and science of mindfulness: Integrating mindfulness into psychology and the helping professions. Washington, DC: American Psychology Press.

Sheldon, K. M., \& Elliot, A. J. (1999). Goal striving, need satisfaction, and longitudinal well- being: The self-concordance model. Journal of Personality and Social Psychology, 76, 482- 497.

Shonin, E., Van Gordon, W., \& Griffiths, M. D. (2013). Mindfulness-based interventions: Towards mindful clinical integration. Frontiers in Psychology, 4. 1-4.

Sin, N. L., \& Lyubomirsky, S. (2009). Enhancing well-being and alleviating depressive symptoms with Positive Psychology Interventions: A practice friendly meta-analysis. Journal of Clinical Psychology, 65, 467-487.

Stevens, C., \& Bryan, A. (2012). Rebranding exercise: there's an app for that. American Journal of Health Promotion, 27(2), 69-70.

Ussher, M., Spatz, A., Copland, C., Nicolaou, A., Cargill, A., Amni-Tabrizi, N., \& McCracken, L. M. (2014). Immediate effects of a brief mindfulness-based body scan on patients with chronic pain. Journal of Behavioural Medicine, 37(1), 127-134.

Watson, D., Clark, L. A., \& Tellegen, A. (1988). Development and validation of brief measures of positive and nega- tive affect: the PANAS scales. Journal of Personality and Social Psychology, 54, 1063-1070.

Webb, T. L., Joseph, J., Yardley, L., \& Michie, S. (2010). Using the Internet to promote health behavior change: A systematic review and meta-analysis of the impact of theoretical basis, use of behavior change techniques, and mode of delivery on efficacy. Journal of Medical Internet Research, 12(1), 97-114.

Wolfenden, L., Brennan, L., Britton, B. I. (2010). Intelligent obesity interventions using Smartphones. Preventive Medicine: An International Journal Devoted to 
Practice and Theory, 51(6), 519- 520.

Zeidan, F., Gordon, N.S., Merchant, J., \& Goolkasian, P. (2010). The Effects of Brief Mindfulness Meditation Training on Experimentally Induced Pain. Journal of Pain, 11(3), 199-209 